\title{
Geometrical Visualization of Indirect Space Vector Modulation for Matrix Converters Operating with Abnormal Supplies
}

\author{
Quanxue Guan ${ }^{1,2}$, Pat Wheeler ${ }^{2}$, Olaf Simon ${ }^{3}$, Quansheng Guan ${ }^{凶}$, Jon Clare ${ }^{2}$ \\ ${ }^{1}$ School of Automation, Guangdong University of Technology, Guangzhou, 510006, China \\ ${ }^{2}$ Department of Electrical and Electronics Engineering, University of Nottingham, Nottingham, NG7 2RD, U.K. \\ ${ }^{3}$ SEW-EURODRIVE GmbH \& Co KG, Bruchsal, D-76642, Germany \\ ${ }^{4}$ School of Electronic and Information Engineering, South China University of Technology, Guangzhou, 510640, China \\ E-mail: eeqshguan@scut.edu.cn
}

\begin{abstract}
Matrix Converters can be sensitive to abnormal supply conditions due to the absence of energy storage elements. This sensitivity can get worse when the Matrix Converters are modulated by a traditional Indirect Space Vector Modulation that assumes the input variables are sinusoidal and balanced. Therefore, this paper proposes a methodology for the modulation of Matrix Converters without requiring any assumption of the input voltages. The method uses a geometric representation based on the Singular Value Decomposition of the switch states to synthesize the rotating vectors of the target duty-cycle matrix. Furthermore, this paper mathematically highlights the factors that have regulate the amplitude of the output voltages and utilizes them to compensate the adverse effect of the abnormal input voltages. Experimental results presented in this paper validate that the proposed method can provide sinusoidal and balanced output currents in the presence of abnormal supply conditions.
\end{abstract}

\section{Introduction}

The matrix converter (MC) is one of the well-known topologies for AC-AC power conversion due to its inherent advantages such as bi-directional power flow, adjustable input power factor and high power density [1-4]. The compactness and potentially long lifetime resulting from the elimination of bulky electrolytic capacitors make the MC and its topology derivations suitable for offshore wind farms [5, 6], solid-state transformers [7, 8] and more-electric-aircraft applications [9-12].

A good implementation of a suitable modulation is fundamental to achieve good performance from any power converter. Many methods have been proposed for the modulation of MCs [13], including the scalar type methods [14] and vectorial type methods [15, 16], to optimize the switching sequence [17], to improve the output quality [18-21], to increase the voltage transfer ratio [22] or to feature the over-modulation capability [23]. Among them, the indirect space vector modulation (ISVM) method is widely used due to its easy implementation [24]. Many derivations of this traditional ISVM assume sinusoidal and balanced supply and load [24, 25]. However, when the input voltage disturbances are not compensated properly, the output voltages may contain unwanted harmonics

Both feedback control and feedforward compensation can be used to counteract the low-frequency undesirable supply voltage fluctuations [25-27]. Many closed-loop control algorithms have been widely reported in literature. However, since the transfer characteristics between the supply voltages and the load currents are nonlinear and complex, it is difficult to design a controller with very good performance under all operating conditions. Therefore, the feedforward method is preferred because it provides faster response and is easier to design and implement. Given that the instantaneous input voltages have to be precisely measured in most MC applications, it is intuitive to use the measured quantities in an open-loop controller to compensate any input distortion [14-16].

The feedforward method can be incorporated into the ISVM technique by modifying the switching functions of either the virtual rectifier or inverter to suppress the input disturbances [28-34]. This is analogous to the pulse width modulation algorithms for traditional back-to-back converters [24]. In [28-30], the rectifier switching functions are regulated appropriately to eliminate the influence of the abnormal supply voltages and generate a constant (on averaged) DC-link voltage. The load side switching functions can then be selected as the same way as in a regular inverter. However, the averaged DC-link current is variable, which can complicate the input current synthesis. If the rectifier switching functions are chosen to be sinusoidal which results in a constant DC-link current but a varying DC-link voltage, the inverter switching functions have to be modified accordingly to maintain sinusoidal and balanced output waveforms [30-33]. It is also possible to regulate both input and output side switching functions synchronously in one step [34]. However, in this case both the DC-link current and voltage are varying, which makes the feedforward compensation more difficult.

The existing modified-ISVM methods discussed above rely on the DC-link concept [24]. They inevitably change the DC-link voltage or current when regulate the switching functions. Unfortunately, it is difficult to derive and calculate the analytical solution for either the transient or the averaged DC-link voltage and/or current, especially when the inputs are abnormal [26]. Further, these ISVM methods decompose the input and output quantities into symmetrical components or harmonics components. Such decompositions might be useful for harmonic analyses but are not necessary for the feedforward compensation and modulation calculation.

The interpretation and implementation complexity discussed above is due to the tradition that modulation derivation is relied on voltage/current space vectors [24] or similar quantities [26] formed by switch states and system input variables together. Whereas, in fact, the switch states present the inner connection of a MC regardless the variations of the input voltages and the output currents. Therefore, it is more applicable and preferable to separately consider the switch states and the system inputs of MCs [35-37].

Investigation into the switch states has therefore become significant in deriving new modulation algorithms for MCs. In this paper the method presented in [36] is extended and experiment results are presented to validate the method. To start with, this paper applies the singular value decomposition (SVD) technique to the space vector transfer functions of switch state matrices rather than the duty-cycle matrices. It is worthy to point out the fact that there are numerous duty-cycle matrix solutions but only limited number of switch state 
solutions to the matrix converter input/output transfer functions. The decomposed matrices have sequential geometrical operations that rotate the vectors of system inputs. This can be used to demonstrate how the MCs manipulate their input voltages and output currents to their counterparts at the corresponding output and input sides, and thus reveal the intrinsic properties of switch states. After representing the decomposed submatrices by their equivalent vectors with the same rotation effects, the space vector modulation (SVM) technique can be adopted to select appropriate switch states and then synthesize them to be the continuously rotating local-averaged reference variables.

The findings presented in this paper provide a new understanding of the modulation process. Accordingly, motivated by counteracting the abnormal input voltages, this work proposes a flexible ISVM algorithm for both direct and indirect MCs. The main contributions of this paper are:

- Geometrical interpretation: A geometrical interpretation for the switch state and the duty cycle matrices of MCs is presented according to the physical meanings of SVD. This provides an understandable visualization for the modulation process.

- Feedforward compensation: It proves mathematically that the modulation index, the amplitudes of the virtual rectifier and virtual inverter switching functions present the same feedforward capability. - Flexibility: The vector representation of the switch states makes the utilization of the SVM technique possible and easy, which leads to improved flexibility in terms of the switch state selection, the duty cycle calculation and the switching sequence arrangement.

\section{Singular Value Decomposition based Indirect Space Vector Modulation Method}

A three-phase-to-three-phase direct MC is shown in Fig. 1(a). Typically, the converter input is a three-phase voltage source because of the nature of the grid and/or LC filter, while the output is a current source since the MC is usually used to feed inductive loads such as motor drives. The MC connects two different types of sources via a $3 \times 3$ switch array. Thus, short circuits between the input lines meanwhile open circuits at each output phase should be prohibited for safe operation.

With the help of the indirect transfer function approach [24], the single-stage MC in Fig. 1(a) can be mathematically divided into a two-stage one as shown in Fig. 1(b) where $p$ and $n$ represent the positive and negative virtual DC bus, while $a, b, c$ the three input phases and $A, B, C$ the three output phases. This is the so-called "Indirect MC" where a current source rectifier (CSR) connects directly to a voltage source inverter (VSI). Due to the existence of the fictitious DC-link, only 21 out of all 27 valid SSMs for direct MCs have equivalent connections in indirect MCs and therefore explored in this work.

For the sake of simplicity, the input/output relationship of the MC system can be expressed in the $\alpha \beta$-coordinates as given below [36],

$$
\begin{gathered}
{\left[\begin{array}{c}
v_{o \alpha} \\
v_{o \beta}
\end{array}\right]=\left[\begin{array}{ll}
S_{1 \alpha} & S_{1 \beta} \\
S_{2 \beta} & S_{2 \alpha}
\end{array}\right]\left[\begin{array}{c}
v_{i \alpha} \\
v_{i \beta}
\end{array}\right]=\mathbf{S}_{x y z}\left[\begin{array}{c}
v_{i \alpha} \\
v_{i \beta}
\end{array}\right],} \\
{\left[\begin{array}{c}
i_{i \alpha} \\
i_{i \beta}
\end{array}\right]=\left[\begin{array}{ll}
S_{1 \alpha} & S_{2 \beta} \\
S_{1 \beta} & S_{2 \alpha}
\end{array}\right]\left[\begin{array}{c}
i_{o \alpha} \\
i_{o \beta}
\end{array}\right]=\mathbf{S}_{x y z}^{T}\left[\begin{array}{c}
i_{o \alpha} \\
i_{o \beta}
\end{array}\right],}
\end{gathered}
$$

where $\mathbf{S}_{x y z}$ is the switch state matrix (SSM), i.e. the space vector represented transfer matrix with $x y z$ standing for the input phases that are linked to the output ones $[36,37]$. For example, when the three output phases $A, B$ and $C$ are connected in turn to the input phases $a, b$ and $b, \mathbf{S}_{x y z}$ can be written as $\mathbf{S}_{a b b}$. The superscript " $T$ " denotes the transpose. Here, the input voltage space vector can be expressed by its column vector as $\vec{v}_{i}=v_{i \alpha}+j v_{i \beta}$. Similarly, the output current space vector is obtained, $\vec{i}_{o}=i_{o \alpha}+j i_{o \beta}$. They together are the system inputs, while the output voltage space vector $\vec{v}_{o}=v_{o \alpha}+j v_{o \beta}$ and the input current space vector $\vec{i}_{i}=i_{i \alpha}+j i_{i \beta}$ are the system output variables.

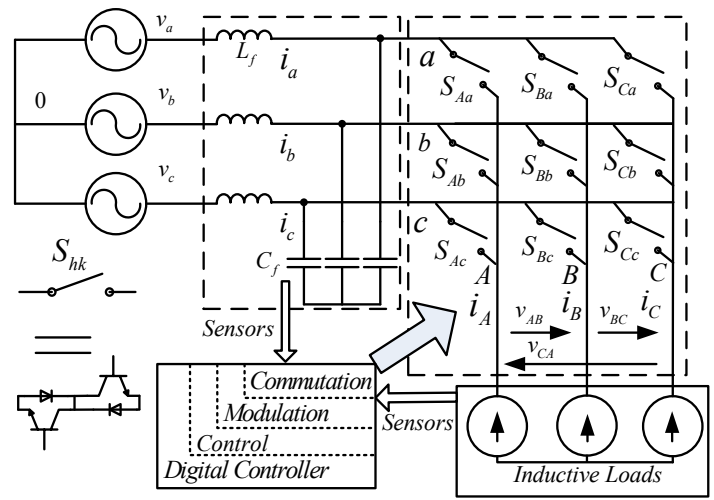

(a)

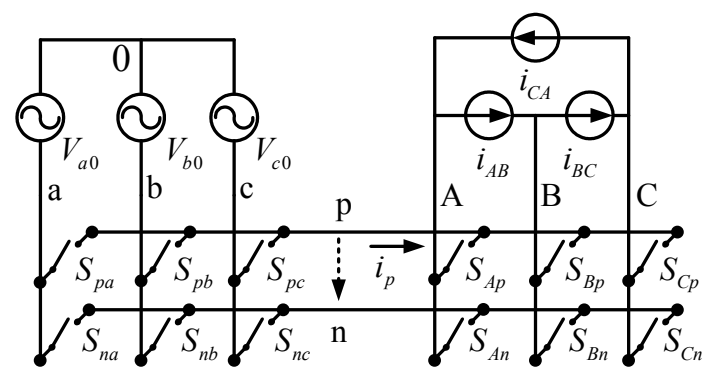

(b)

Fig. 1: (a) The typical topology of a three-phase direct matrix converter [36] and (b) its simplified two-stage indirect derivation [24].

The existing modulation methods were derived based on the space vectors or similar quantities that are the products of switch states and systems inputs [14-16, 24-34]. For example, in [24] the line-line voltage space vector can be defined by

$$
\vec{v}_{o l}=\frac{2}{3}\left(v_{A B}+v_{B C} \cdot e^{120^{\circ}}+v_{C A} \cdot e^{240^{\circ}}\right) .
$$

In abnormal input voltage scenarios, the resulting discrete space vectors are with varying amplitudes. This violates the assumption of constant amplitude vectors as that in regular PWM inverters, and makes the modulation process intricate [26]. To avoid the varying vector problem and to explore the inherent features of the $\mathrm{MC}$, this work derives a novel but simple ISVM algorithm starting from analyzing the valid SSMs only rather than their corresponding voltage and current space vectors.

\subsection{Singular Value Decomposition of Switch States}

From the matrix theory, all SSMs have transforming effects between the system input and output space vectors. In order to reveal such transformation, the SVD technique is applied to the matrix $\mathbf{S}_{x y z}$, on account of the similar geometric operations between SVD and $\mathbf{S}_{x y z}$. This results in a set of multiplied matrices, representing as $\mathbf{S}_{x y z}=$ $\mathbf{U} * \mathbf{D} * \mathbf{V}^{T}$, where both $\mathbf{U}$ and $\mathbf{V}$ are unitary matrices, while $\mathbf{D}$ is a diagonal matrix [36-39]. Note that the SVD technique used in this work is not for principle component analyses but to provide insights to the intrinsic properties of the SSMs of MCs and categorize them into groups, with the help of the geometrical meaning of SVD.

All 27 SSMs have their own decompositions which are listed in Table 1 (where $x x x$ means $a a a, b b b$ or $c c c$ ) [36]. They have a series of sequential effects on the input vectors in (1). Within them are the Type II Switch States (T2SS) that connects two and only two output lines to one common input phase. The unitary matrices $\mathbf{U}$ and $\mathbf{V}$ retrogress to rotation matrices while the secondary gain in $\mathbf{D}$ is always zero. 
Table 1 Singular Value Decomposition Results of the Switch States [36]

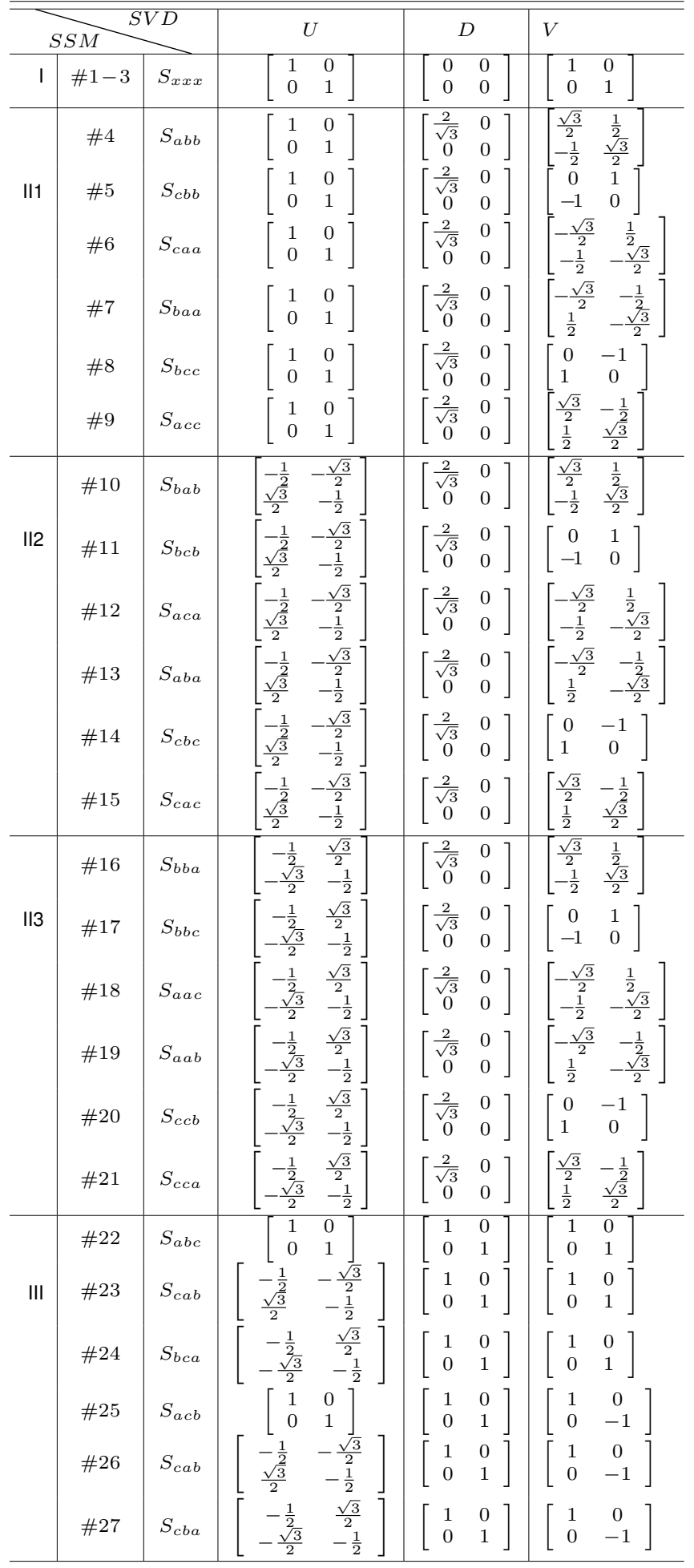

Take $S_{a c c}$ as an example, shown in Fig. 2. Its expression in $\alpha \beta$ plane can be calculated, and decomposed:

$$
\mathrm{S}_{\mathrm{acc}}=\left[\begin{array}{cc}
1 & \frac{\sqrt{3}}{3} \\
0 & 0
\end{array}\right]=\left[\begin{array}{ll}
1 & 0 \\
0 & 1
\end{array}\right]\left[\begin{array}{cc}
\frac{2}{\sqrt{3}} & 0 \\
0 & 0
\end{array}\right]\left[\begin{array}{cc}
\frac{\sqrt{3}}{2} & -\frac{1}{2} \\
\frac{1}{2} & \frac{\sqrt{3}}{2}
\end{array}\right]^{T} .
$$

According to the geometric meaning of the SVD technique, the matrix $\mathbf{V}^{T}$ of $S_{a c c}$ rotates any input column vector in (1) by $-30^{\circ}$, or equivalently $\mathbf{V}$ rotates the input coordinate by $30^{\circ}$. Note that the transpose of a rotation matrix effectively rotates by a negative angle. Then, the matrix $\mathbf{D}$ scales the $d$-axis of the resultant vector

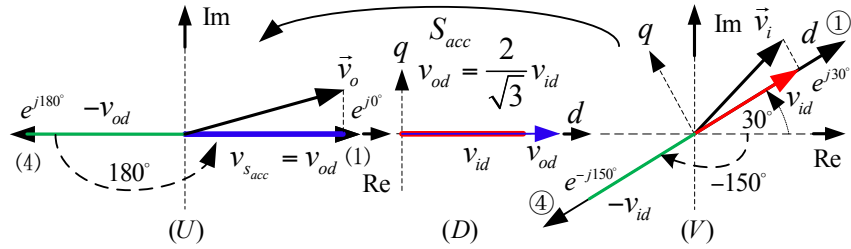

Fig. 2: The geometrical meanings of the SVD upon the switch state $S_{a c c}$.

by $2 / \sqrt{3}$ while zeroes the $q$-axis component. Finally, the matrix $\mathbf{U}$ rotates the intermediate quantity $\mathbf{D V}^{T} \vec{v}_{i}$ by $0^{\circ}$ to obtain an output vector. $S_{a c c}^{T}$ has a similar operation but in reverse sequence on the current relation.

The switch states $S_{a c c}$ may have other SVD results, like

$$
\mathrm{S}_{\mathrm{acc}}=\left[\begin{array}{cc}
1 & \frac{\sqrt{3}}{3} \\
0 & 0
\end{array}\right]=\left[\begin{array}{cc}
-1 & 0 \\
0 & -1
\end{array}\right]\left[\begin{array}{cc}
\frac{2}{\sqrt{3}} & 0 \\
0 & 0
\end{array}\right]\left[\begin{array}{cc}
-\frac{\sqrt{3}}{2} & \frac{1}{2} \\
-\frac{1}{2} & -\frac{\sqrt{3}}{2}
\end{array}\right]^{T} \text {. }
$$

From the geometric perspective, the matrices in (5) first rotates the input column vector in (1) by $150^{\circ}$ or in other word, rotates the coordinate system by clockwise $150^{\circ}$, and subsequently scales the $d$-axis component and rotates the resultant vector by $180^{\circ}$ in sequence. The input vector $\vec{v}_{i}$ is transformed to the same position as (4) does. Actually, different SVD results for each matrix $\mathbf{S}_{x y z}$ equally describe the same linear coordinate transformation.

The geometric effects of the rotation matrices Us and Vs in the SVD results of T2SS can be represented by their equivalent vectors with the same rotation angles. For instance,

$$
\left[\begin{array}{cc}
\cos \vartheta & -\sin \vartheta \\
\sin \vartheta & \cos \vartheta
\end{array}\right] \Leftrightarrow e^{j \vartheta}
$$

All these rotation vectors are with unity amplitude and fixed angles. They form two hexagons and evenly divide them into six sectors respectively, as shown in Fig. 3(a). However, they are distinct from their voltage and current counterparts in the existing ISVM algorithms [14-16, 24-34] where the output line voltage and the input current space vectors depend on the products of the SSMs and the system inputs, and hence are varying in terms of length, as shown in Fig. 3(b). According to (3) and similar expressions to the current quantities, the resulting discrete vectors are determined by not only the switch states but also the DC-link voltage or current. Within one the switching cycle, since the DC-link voltage is rectified by the CSR, using two or more switching states, and thus has different transient values, e.g. $v_{a b}$ and $v_{a c}$ (referring to vectors $I_{6}(a, b)$ and $I_{1}(a, c)$ in Fig. 3(b)) [26]. This corresponds to varying vector amplitudes as shown by the grey area of the hexagons in Fig. 3(b). It is true even when systems inputs are sinusoidal and balanced. Under fluctuated input conditions, the deviations become more complex, where the amplitudes of the local-averaged hexagons in dot-dash line are not constant since the averaged DC-link voltage and current are variable. This phenomenon makes the vector syntheses intricate. On the contrary, our method provides a simpler and easier base to derive new modulation algorithms since both the amplitude and angle of the used vectors are constant.

The T2SS are also summarized in Table 2 by matching the rotation angles between the decomposed $\mathbf{U s}$ and $\mathbf{V s}$ and their equivalent rotation vectors in related hexagons of Fig. 3. For instance, the vector directing to pointer (1) in the left hexagon corresponds to the six T2SS in the first line of Table 2, while the one synchronously directing to pointers (1) and (1) in both hexagons is the SSM $\mathbf{S}_{a c c}$. The fact that both entries of (1)(1) and (4)(4) in Table 2 are the SSM S acc $_{a c}$ can be explained by (4) and (5) in the example given above. In the rest of this paper, we use the same symbols to describe the rotation vectors in Fig. 3 (e.g. vector (1)) and their corresponding decomposed rotation matrices (e.g. column (1) in Table 2). This notation method brings extra benefits for the switch state selection and the 


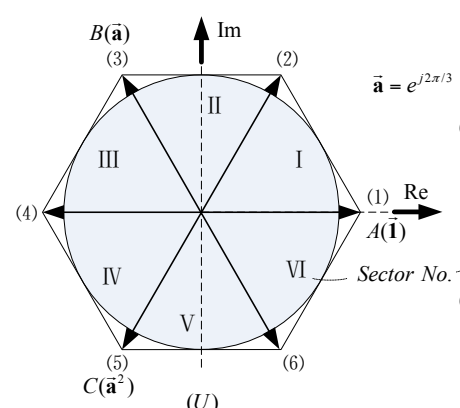

$(U)$

(a)

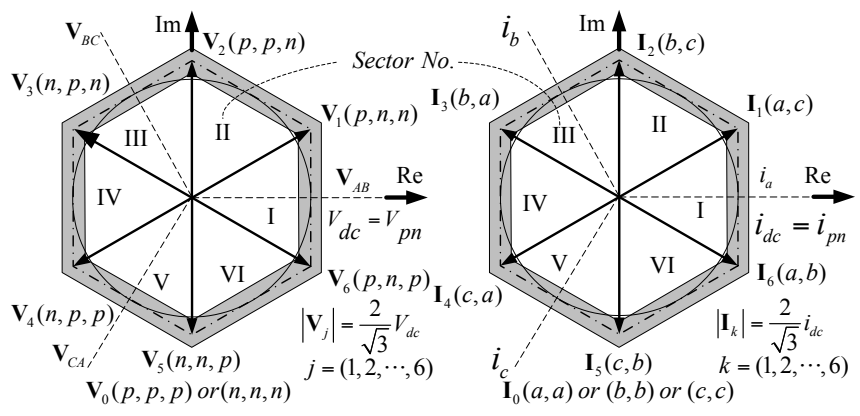

(b)

Fig. 3: (a) The new proposed output (left) and input (right) hexagons formed by the equivalent vectors of the Type II Switch States [36]. (b) Virtual inverter output line voltage hexagon (left) and virtual rectifier input current hexagon (right) in the traditional ISVM algorithms for matrix converters [14-16, 24-34].

Table 2 Type II Switch States Summarized According to Their Angles

\begin{tabular}{c|cccccc}
\hline $\mathbf{U}$ & (1) & (2) & (3) & (4) & (5) & (6) \\
\hline$(1)$ & $S_{a c c}$ & $S_{b c c}$ & $S_{b a a}$ & $S_{c a a}$ & $S_{c b b}$ & $S_{a b b}$ \\
$(2)$ & $S_{a a c}$ & $S_{b b c}$ & $S_{b b a}$ & $S_{c c a}$ & $S_{c c b}$ & $S_{a a b}$ \\
$(3)$ & $S_{c a c}$ & $S_{c b c}$ & $S_{a b a}$ & $S_{a c a}$ & $S_{b c b}$ & $S_{b a b}$ \\
$(4)$ & $S_{c a a}$ & $S_{c b b}$ & $S_{a b b}$ & $S_{a c c}$ & $S_{b c c}$ & $S_{b a a}$ \\
$(5)$ & $S_{c c a}$ & $S_{c c b}$ & $S_{a a b}$ & $S_{a a c}$ & $S_{b b c}$ & $S_{b b a}$ \\
$(6)$ & $S_{a c a}$ & $S_{b c b}$ & $S_{b a b}$ & $S_{c a c}$ & $S_{c b c}$ & $S_{a b a}$ \\
\hline
\end{tabular}

commutation arrangement which will be shown later in Section 2.3 and Section 3.2.

\subsection{Geometrical Interpretation for the Duty-cycle Matrix}

Time-averaging the valid SSMs will obtain the duty-cycle matrix whose representation in $\alpha \beta$ reference frame has the similar SVD structure as given below [36],

$$
\overline{\mathbf{U}} * \overline{\mathbf{D}} * \overline{\mathbf{V}}^{T}=\left[\begin{array}{cc}
\cos \alpha & -\sin \alpha \\
\sin \alpha & \cos \alpha
\end{array}\right]\left[\begin{array}{cc}
g_{d} & 0 \\
0 & g_{q}
\end{array}\right]\left[\begin{array}{cc}
\cos \theta & -\sin \theta \\
\sin \theta & \cos \theta
\end{array}\right]^{T},
$$

where matrices $\overline{\mathbf{U}}, \overline{\mathbf{D}}$ and $\overline{\mathbf{V}}$ are the local-averaged values of $\mathbf{U}$, $\mathbf{D}$, and $\mathbf{V}$ according to the duty cycles of the SSMs used in one sampling period. Both $\mathbf{V}$ and $\mathbf{U}$ are rotation matrices, so be their counterparts $\overline{\mathbf{V}}$ and $\overline{\mathbf{U}} . g_{d}$ and $g_{q}$ are the two local-average gain in the diagonal matrix $\overline{\mathbf{D}}$. Equation (7) satisfies the system equations (1) and (2) simultaneously and thus is a solution to the modulation matrix. In (7), $\overline{\mathbf{V}}^{T}$ rotates the input side quantities by $-\theta$ while $\overline{\mathbf{U}}^{T}$ rotates the output vectors by $-\alpha$. This also equals to that $\overline{\mathbf{V}}$ and $\overline{\mathbf{U}}$ rotate the natural reference frame by $\theta$ and $\alpha$ respectively to form two rotating reference frames. In these rotating frames, the voltage and current space vectors at the input and output sides of the MC system can be decomposed, and their relations are determined by

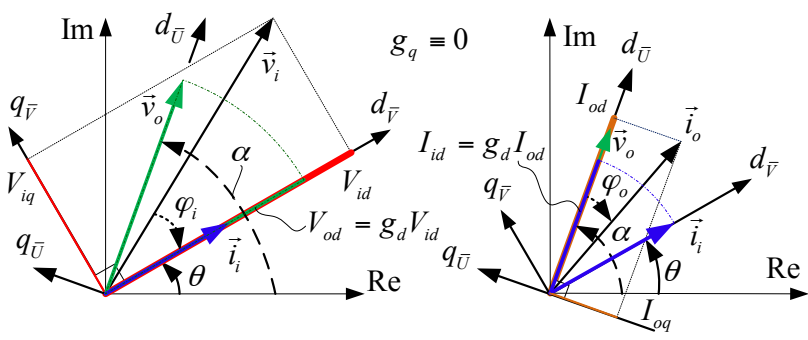

Fig. 4: The decomposition and transformation of the input/output voltage and current space vectors between two rotating reference frames.

the diagonal matrix $\overline{\mathbf{D}}$. Since the T2SS listed in Table 1 [36] share the same zero secondary entries in the diagonal matrices, their corresponding local-averaged value $g_{q}$ in $\overline{\mathbf{D}}$ always equals zero. This brings several benefits for implementing the modulation process.

Firstly, the voltage and current relations between two reference frames can be expressed below,

$$
V_{o}=g_{d} V_{i d}, \quad I_{i}=g_{d} I_{o d}
$$

where $V_{o}$ and $I_{i}$ are the amplitudes of the output voltage space vector $\vec{v}_{O}$ and the input current space vector $\vec{i}_{i}$ respectively. They are constructed from parts of the input voltage and output current. Secondly, the $q$-axis component of $\vec{v}_{o}$, in the reference frame constructed by the matrix $\overline{\mathbf{U}}$, and the $q$-axis component of $\vec{i}_{i}$, in the reference frame constructed by the matrix $\overline{\mathbf{V}}$, are always zero because of $g_{q} \equiv 0$ in (7). That means $\vec{v}_{O}$ is always located at the rotation vector $\mathbf{U}$, while $\vec{i}_{i}$ is always placed at the rotation vector $\overline{\mathbf{V}}$. As a consequence, the output frequency and the input power factor can be controlled by the angles $\alpha$ and $\theta$.

The SVD renders the modulation process a clear physical meaning as shown in Fig. 4. From the geometric perspective, the rotating reference frames that $\overline{\mathbf{V}}$ and $\overline{\mathbf{U}}$ construct are the input and output synchronous reference frame rotating along with $\vec{i}_{i}$ and $\vec{v}_{o}$. In both reference frames, the input and output frequencies are counteracted. For example, the vector $\vec{i}_{i}$ in the stationary reference frame now becomes a scalar quantity $\vec{i}_{i} e^{-j \theta}=\mathrm{I}_{i}$ in the input synchronous reference frame. The input voltage and output current space vectors are decomposed into $d$ - and $q$-axis components in their corresponding synchronous reference frames given the existence of input and output power displacements. But only their $d$-axis components are scaled and rotated back to the stationary reference frame, while the $q$-axis components are shrank to zero. Although two synchronous reference frames rotate at different frequencies, the relations of the decomposed components are determined only by the diagonal matrix $\overline{\mathbf{D}}$ or the $g_{d}$. Therefore, the parameters $\alpha, \theta$ and $g_{d}$ form the three free variables to synthesize any desired amplitude and frequency of the output voltage and input phase displacement factor, while the amplitude of the input current is determined by the active power balance.

The rank reduction in $\overline{\mathbf{D}}$ makes the indirect MC similar to backto-back converters and makes the quantity relationships at the virtual intermediate stage scalar regardless of the angle difference between two rotating frames in Fig. 4 as if there exists a the DC-link. It is worth noting that in Table 1 there remain six SSMs, connecting each output phase to one different input line, being valid for direct MCs but not indirect MCs. They are the Type III Switch States which have non-zero diagonal entries in $\overline{\mathbf{D}}$ submatrices and thus have the rank of 2. Also, they preserve the unitary features in either $\overline{\mathbf{U}}$ or $\overline{\mathbf{V}}$ which has a determinant +1 or -1 [37]. This mathematically proves that when only using the SSMs in Table 2 the indirect MC is an incomplete equivalence of the direct MC which uses all 27 valid SSMs. 

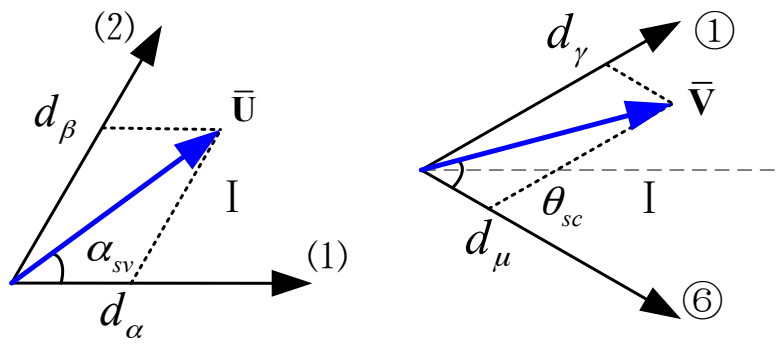

Fig. 5: Examples of space vector syntheses using two adjacent vectors in the output (left) and input (right) hexagons.

\subsection{Space Vector Synthesis}

Since the used SSMs and their local-averaged duty-cycle matrices share similar SVD results that can be represented in vector formation, it is easy to synthesize the latter using the former. Once the output frequency and the input power factor are given or determined by the outer control loop, the rotating vectors $\overline{\mathbf{U}}$ and $\overline{\mathbf{V}}$ can be portrayed in Fig. 3. Then they are assumed invariant during specific sampling period and can be synthesized by using the well-known SVM method [24].

Without loss of generality, $\overline{\mathbf{U}}$ and $\overline{\mathbf{V}}$ are assumed to locate in Sector I. They can be approximated by two groups of adjacent vectors respectively to maximize their amplitudes. As shown in Fig. 5, the duty cycle for each active vector can be determined as follows [36],

$$
\begin{aligned}
& d_{\alpha}=\frac{2}{\sqrt{3}} \cdot|\overline{\mathbf{U}}| \cdot \sin \left(60^{\circ}-\alpha_{s v}\right), d_{\beta}=\frac{2}{\sqrt{3}} \cdot|\overline{\mathbf{U}}| \cdot \sin \alpha_{s v}, \\
& d_{\mu}=\frac{2}{\sqrt{3}} \cdot|\overline{\mathbf{V}}| \cdot \sin \left(60^{\circ}-\theta_{s c}\right), d_{\gamma}=\frac{2}{\sqrt{3}} \cdot|\overline{\mathbf{V}}| \cdot \sin \theta_{s c}
\end{aligned}
$$

where $\alpha_{s v}$ and $\theta_{s c}$ are the angles of the two reference vectors with respect to the beginning of their sectors. $|\overline{\mathbf{U}}|$ and $|\overline{\mathbf{V}}|$ are the amplitudes of $\overline{\mathbf{U}}$ and $\overline{\mathbf{V}}$ respectively. The vectors pointing to (1), (2) and (6), (1) in Fig. 3 are used. They correspond to the SSMs in the lines (1), (2) and columns (6), (1) in Table 2, i.e. $S_{a b b}, S_{a c c}, S_{a a b}$ and $S_{\text {aac. }}$. The first two SSMs have the same $\mathbf{U}$ and $\mathbf{D}$, and thus approximate Vector $\overline{\mathbf{V}}$ at the input hexagon meanwhile Vector (1) at the output hexagon with duty cycles being proportional according to $d_{\alpha}$ and ${ }_{\beta}$ in (9). The latter two SSMs approximate the same Vector $\overline{\mathbf{V}}$ at the input side but Vector (2) at the output with duty cycles being proportional according to $d_{\mu}$ and $d_{\gamma}$ in (10). Then, with the same Vector $\overline{\mathbf{V}}$, to synthesize $\overline{\mathbf{V}}$ and $\overline{\mathbf{U}}$ synchronously, the duty cycles for the four selected SSMs have to be proportional, as given below,

$$
\begin{aligned}
& \left(S_{a b b}:\right) d_{\alpha \mu}=m \cdot d_{\alpha} \cdot d_{\mu},\left(S_{a c c}:\right) d_{\alpha \gamma}=m \cdot d_{\alpha} \cdot d_{\gamma}, \\
& \left(S_{a a b}:\right) d_{\beta \mu}=m \cdot d_{\beta} \cdot d_{\mu},\left(S_{a a c}:\right) d_{\beta \gamma}=m \cdot d_{\beta} \cdot d_{\gamma},
\end{aligned}
$$

where the modulation index $m$ is defined to regulate each duty cycle non-negative and their sum less than unity. The switch states used in this example are added in parentheses to the above formulae. It is important to note that the addition of vectors can be interpreted according to the addition of matrices because they have the same $\mathbf{D}$ or $\overline{\mathbf{V}}$ or $\overline{\mathbf{U}}$ in the SVD results.

The the modulation index has two functions. Except for the total duty-cycle regulation, $m$ will also influence the averaged $d$-axis gain of $\overline{\mathbf{D}}$ that is governed by:

$$
g_{d}=m \cdot|\overline{\mathbf{U}}| \cdot \sigma_{d} \cdot|\overline{\mathbf{V}}| \text {, }
$$

where $\sigma_{d}$ is the primary diagonal entry of $\mathbf{D}$, i.e. $2 / \sqrt{3}$. According to (12), $m,|\overline{\mathbf{U}}|$ and $|\overline{\mathbf{V}}|$ can be used to regulate $g_{d}$. However, the determinants of the desired $\overline{\mathbf{U}}$ and $\overline{\mathbf{V}}$ in (7) are unity but the actual amplitudes according to (9) and (10) are not. To make up the desired $\overline{\mathbf{U}}, \overline{\mathbf{D}}$ and $\overline{\mathbf{V}}$, the synthesized lengths of $\overline{\mathbf{U}}$ and $\overline{\mathbf{V}}$ are adjusted so that

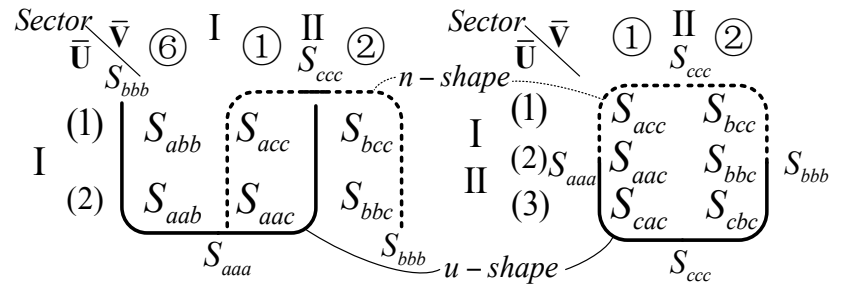

Fig. 6: Switching pattern arrangements when using the most adjacent vectors.

the effective duty cycles are unchanged before and after this adjustment. In this work, $|\overline{\mathbf{U}}|$ and $|\overline{\mathbf{V}}|$ are chose as the maximum radii of the inscribed circles of their hexagons, i.e. $\sqrt{3} / 2$ for the adjacent vector synthesis. Then $g_{d}=\sqrt{3} \mathrm{~m} / 2$. Now that $g_{d}$ is subject to $m$ only, Eq. (11) can be rewritten,

$$
\begin{aligned}
& d_{\alpha \mu}=m \cdot \sin \left(60^{\circ}-\alpha_{s v}\right) \sin \left(60^{\circ}-\theta_{s c}\right), \\
& d_{\alpha \gamma}=m \cdot \sin \left(60^{\circ}-\alpha_{s v}\right) \sin \left(\theta_{s c}\right), \\
& d_{\beta \mu}=m \cdot \sin \left(\alpha_{s v}\right) \sin \left(60^{\circ}-\theta_{s c}\right), \\
& d_{\beta \gamma}=m \cdot \sin \left(\alpha_{s v}\right) \sin \left(\theta_{s c}\right) .
\end{aligned}
$$

The remaining time interval within the sampling period is the duty cycle $d_{0}$ for the zero vectors formed by the T1SS,

$$
d_{0}=1-d_{\alpha \mu}-d_{\beta \mu}-d_{\alpha \gamma}-d_{\beta \gamma} .
$$

To complete the modulation process, the switching sequence among different switch states has to be arranged appropriately. The idea presented in $[31,35]$ is adopted and shown in Fig. 6. When the sum of two sector numbers is even, an " $u$ " shape pattern is utilized as the switching sequence. When this sum is odd, an " $n$ " shape pattern is employed. T1SS can be placed at the beginning, the center or the end of the switching pattern. For the example discussed in this section, the first half of a double-sided switching sequence is arranged as $\left(S_{b b b}\right) \rightarrow S_{a b b} \rightarrow S_{a a b} \rightarrow\left(S_{a a a}\right) \rightarrow$ $S_{a a c} \rightarrow S_{a c c} \rightarrow\left(S_{c c c}\right)$ where one to three T1SS within the parentheses can be used. The flexibility of arranging the switching pattern in this way are usually exploited to trade off the performance criteria among the commutation number, the switching loss, the output current ripple and the common-mode voltage.

\subsection{Feedforward Compensation}

A simple method to determine $m$ is to set it to a constant, which leads to the same result as in [24]. Unfortunately, since the system input and output are coupled, the input distortion may deteriorate the output performance. For constant $m$, the low frequency harmonics of the input voltages can be transferred to the output voltages. Similarly, harmonic contents might also contain in the output side, then transfer back to the input currents, and distort the input voltages when the input filters are considered. The distorted input voltages make the output performance even worse. This is undesirable.

According to (8), the output voltage is made only from the $d$-axis component of the input voltages. Therefore, the voltage transfer ratio (VTR) $q$ can be described by

$$
q=\frac{\left|\vec{v}_{o, r e f}\right|}{\left|\vec{v}_{i}\right|}=\frac{V_{o d} \cdot \cos \varphi_{i}}{V_{i d}}=g_{d} \cdot \cos \varphi_{i}
$$

where $\varphi_{i}$ is the input displacement angle. From (12) and (15), the modulation index $m$ can be determined as shown below:

$$
m=\frac{2}{\sqrt{3}} \cdot \frac{\left|\vec{v}_{o, r e f}\right|}{\left|\vec{v}_{i}\right| \cos \varphi_{i}} .
$$

The only limitation for $m$ is to maintain all duty cycles in (13) and (14) non-negative. According to (13) and (16), the angle of the input 


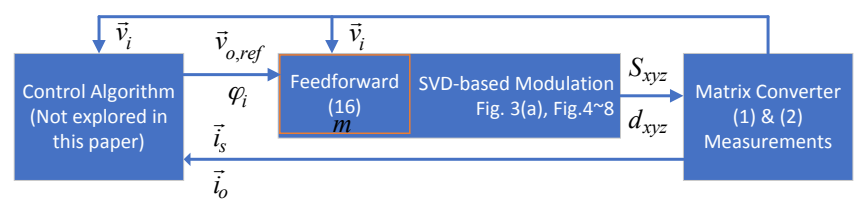

Fig. 7: Relationship between the proposed SVD-based modulation and other control algorithms.

voltage space vector does not engaged in the modulation calculation, but it affects the input displacement factor and thereby the output regulation capacity.

Substituting (16) into (13)-(15) leads to

$$
\vec{v}_{o}(t)=\vec{v}_{o, r e f}(t)
$$

Therefore, once the input voltages are measured and the input power factor is specified, the modulation index can be adjusted accordingly to compensate the abnormal input variations and to maintain the amplitude of the desired output voltages. By this way, low-frequency harmonics in the input voltages can be eliminated from the output waveforms as long as the condition for $m$ is satisfied.

The relationship between the proposed SVD-based space vector modulation and other control algorithms can be shown in Fig. 7.

\section{Remarks and Comparisons}

\subsection{Maximum Feedforward Compensation Capability}

As has been described in Section 2.2, the proposed ISVM can approach the output voltage at the spectra lower than the cut-off frequency of the filters. In other word, the proposed method is capable to suppress the low order harmonics within such spectrum band. One premise for this is that the modulation index has to be in the linear modulation zone. Given the definitions of $\alpha_{s v}$ and $\theta_{s c}$ in (13) and (14), a conservative range for $m$ is $[0,1]$ if sinusoidal and balanced output voltages are required. This means the maximum VTR in the example shown in the previous section is $(\sqrt{3} / 2) \cos \varphi_{i}$ according to (16). It also means the maximum attainable space vector trajectory has to be within the inscribed circle of polygons formed by the used static vectors. Substituting the range of the modulation index $m$ in (16) leads to the following condition,

$$
\left|\vec{v}_{o}\right| \leq \frac{\sqrt{3}}{2}\left|\vec{v}_{i}\right|_{\min } \cos \varphi_{i}
$$

where $\left|\vec{v}_{i}\right|_{\min }$ is the minimum amplitude of $\vec{v}_{i}$.

For distorted input voltages, $\left|\vec{v}_{i}\right|_{\min }$ might not be formulated explicitly, whereas for sinusoidal but unbalanced input voltages, it can be expressed as the subtraction of the positive and negative sequence components, i.e. $\left|\vec{v}_{i}\right|_{\min }=V_{i p}-V_{i n}$. If the modulation index goes into the over-modulation zone, the output voltage is distorted even under ideal system input condition. However, the condition in (18) is also conservative. In the DSP implementation, a sentence of code is inserted to check whether or not the duty-cycle $d_{0}$ goes to negative, so that the linear- or over-modulation zone is detected.

Another premise for fully compensation is the accuracy of the input voltage measurement. According to (16), any filtering manipulation or inaccurately measurement on the input voltage will weaken the modulation compensation capability.

\subsection{Flexibility in Switch State Selection and Commutation}

Apart from two adjacent vectors, two nearest vectors with $120^{\circ}$ phase shift can also be used to synthesize the reference vectors in either the output hexagon or the input hexagon, as shown in Fig. 8. Take that $\overline{\mathbf{U}}$ is synthesized by two adjacent vectors while $\overline{\mathbf{V}}$ is made by the two nearest vectors with $120^{\circ}$ phase shift. The duty cycles for

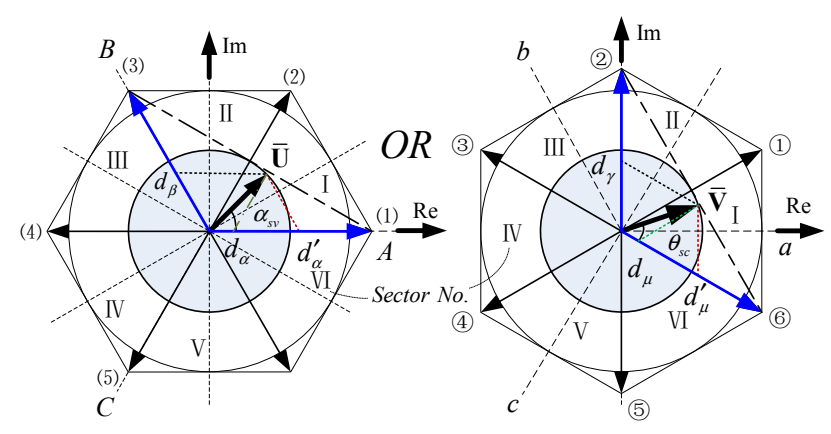

Fig. 8: Space vector syntheses using two vectors with $120^{\circ}$ phase shift.

$\overline{\mathbf{U}}$ can be calculated with (9), but for $\overline{\mathbf{V}}$ (refer to the right hexagon in Fig. 8), equations in (10) are changed to:

$$
d_{\mu}{ }^{\prime}=\frac{2}{\sqrt{3}}|\overline{\mathbf{V}}| \sin \left(120^{\circ}-\theta_{s c}\right), d_{\gamma}=\frac{2}{\sqrt{3}}|\overline{\mathbf{V}}| \sin \theta_{s c} .
$$

Here $\theta_{s c}$ belongs to $\left(30^{\circ}, 90^{\circ}\right)$. The duty-cycle $d_{\mu}{ }^{\prime}\left(=d_{\mu}+d_{\gamma}\right)$ in (19) is much larger than $d_{\mu}$ in in (10). Therefore, using the nearest two vectors with $120^{\circ}$ phase shift in one hexagon can partly alleviate the narrow pulse problem.

However, this method has a limit on the VTR range. Since the maximum length of the corresponding $\overline{\mathbf{V}}$ decreases from $\sqrt{3} / 2$ to $1 / 2, g_{d}$ becomes $m / 2$ according to (12). Substitute it in (15) and recalculate the result for the VTR, then the modulation index $m$ to offset the input voltage is changed to,

$$
m=\frac{2\left|\vec{v}_{o, r e f}\right|}{\left|\vec{v}_{i}\right| \cos \varphi_{i}} .
$$

Similar to the case in Section 2.3, the SSMs in lines (1), (2) and columns (6), (2) in Table 2, i.e. $S_{a b b}, S_{a a b}, S_{b c c}$ and $S_{b b c}$ are selected. The switches states $S_{a a c}$ and $S_{a c c}$ of the four selected SSMs in the previous example are replaced by $S_{b b c}$ and $S_{b c c}$ in this case. Their duty cycles can be calculated accordingly,

$$
\begin{aligned}
& d_{\alpha \mu}{ }^{\prime}=\frac{2}{\sqrt{3}} \frac{\left|\vec{v}_{o}\right|}{\left|\vec{v}_{i}\right| \cos \varphi_{i}} \sin \left(60^{\circ}-\alpha_{s v}\right) \sin \left(120^{\circ}-\theta_{s c}\right), \\
& d_{\beta \mu}{ }^{\prime}=\frac{2}{\sqrt{3}} \frac{\left|\vec{v}_{o}\right|}{\left|\vec{v}_{i}\right| \cos \varphi_{i}} \sin \left(\alpha_{s v}\right) \sin \left(120^{\circ}-\theta_{s c}\right), \\
& d_{\alpha \gamma}=\frac{2}{\sqrt{3}} \frac{\left|\vec{v}_{o}\right|}{\left|\vec{v}_{i}\right| \cos \varphi_{i}} \sin \left(60^{\circ}-\alpha_{s v}\right) \sin \left(\theta_{s c}\right), \\
& d_{\beta \gamma}=\frac{2}{\sqrt{3}} \frac{\left|\vec{v}_{o}\right|}{\left|\vec{v}_{i}\right| \cos \varphi_{i}} \sin \left(\alpha_{s v}\right) \sin \left(\theta_{s c}\right) .
\end{aligned}
$$

They have the similar forms as those in (13) if $m$ is substituted by the value in (16). The complement duty-cycle for the T1SSe is given below,

$$
d_{0}=1-d_{\alpha \mu}{ }^{\prime}-d_{\beta \mu}{ }^{\prime}-d_{\alpha \gamma}-d_{\beta \gamma} .
$$

In a similar way, the switching sequence can be arranged as shown in Fig. 9(a). T1SS $S_{b b b}$ can be chose and placed in the switching sequence during $d_{0}$, so that half of the double-sided switching pattern is $S_{a a b} \rightarrow S_{a b b} \rightarrow S_{b b b} \rightarrow S_{b b c} \rightarrow S_{b c c}$. Given that all the duty cycles should be non-negative, the maximum VTR is $\cos \varphi_{i} / 2$, with $m$ ranging from 0 to 1 .

Likewise, the proposed SVD-based ISVM method can synthesize the reference vectors $\overline{\mathbf{U}}$ or $\overline{\mathbf{V}}$ by using nearest three vectors as shown in Fig. 10(a), which is similar to the idea for back-to-back converters in $[40,41]$ and leads to the same methods in [19]. Besides, the three vectors that are not the nearest vectors adjacent to the desired reference can be used, as shown in Fig. 10(b) and discussed in [37].

\subsection{Comparison with Other Widely Used Modulation Methods}

While $\overline{\mathbf{U}}$ and $\overline{\mathbf{V}}$ are responsible for regulating the directions of output voltage and input current space vectors, their lengths can take 


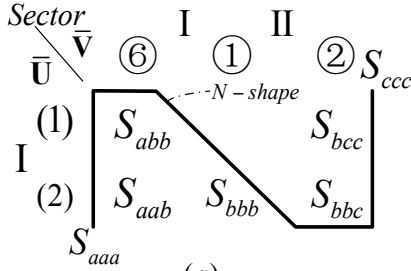

(a)

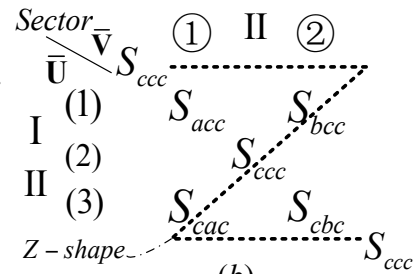

(b)
Fig. 9: Switching patterns: either (a) the input side vector or (b) the output side vector is synthesized by two nearest vectors with $120^{\circ}$ phase shift, while the other side vector is synthesized by two adjacent vectors.

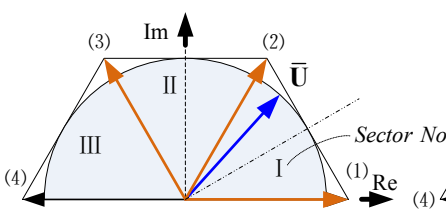

(a)

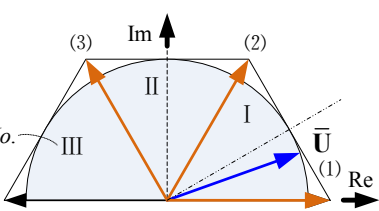

(b)
Fig. 10: Reference vector syntheses using (a) the nearest three vectors or (b) the non-nearest three vectors.

part in adjusting the amplitude of the output voltages, according to (12) and (15). When $|\overline{\mathbf{U}}|$ is assumed constant, adjusting $m$ and $|\overline{\mathbf{V}}|$ results in the same effect of source side modulation in [28-30]. Similarly, adjusting $m$ and $|\overline{\mathbf{U}}|$ under the condition of constant $|\overline{\mathbf{V}}|$ leads to the same as that in load side compensation [30-33]. If $m,|\overline{\mathbf{U}}|$ and $|\overline{\mathbf{V}}|$ are all tuned, the proposed method get the same method as in [34].

One major difference between the proposed and the existing ISVMs lies in the versatility on choosing the active hexagon. To compensate the input voltage abnormalities, the proposed ISVM can choose $|\overline{\mathbf{U}}|$ in one sampling period while $|\overline{\mathbf{V}}|$ in another, and vice versa [37]. But most existing methods in [28-33] modified only the switching functions in one side, either the rectifier side or the inverter side, in every sampling period. Another obvious difference is the proposed ISVM technique calculates the duty cycles using the measured input voltages rather than their decomposed components. This reduces the computation overhead since no decomposition on the input voltage space vector is required in this work. Furthermore, in terms of both the implementation and interpretation aspects, the proposed ISVM algorithm does not count upon the DC-link. This further simplifies the modulation process.

In other generalized modulation methods, the zero vector in [15] and the zero matrix in $[16,42]$ and the translation matrix in [14], which are the different representations of duty cycle $d_{0}$ for the T1SS, are complex and difficult to be calculated. By contrast, the proposed method calculates the $d_{0}$ with less effort and determines the corresponding zero sequence voltage automatically by the SVM technique.

\section{Experimental Results}

In order to verify the effectiveness of the proposed ISVM algorithm, experimental tests are carried out on our laboratory prototype, as shown in Fig. 11. A three-phase inductor and a three-phase resistor are used as the inductive load for the MC. The system parameters are given in Table 3. For simplicity, the modulation strategy sets the input displacement factor to be unity. The distorted and unbalanced input voltages can be emulated by the three-phase programmable voltage source Chroma 61511. The ISVM algorithms are implemented in DSP to calculate the duty cycle for each selected active switch state. Such information will be transmitted via the external

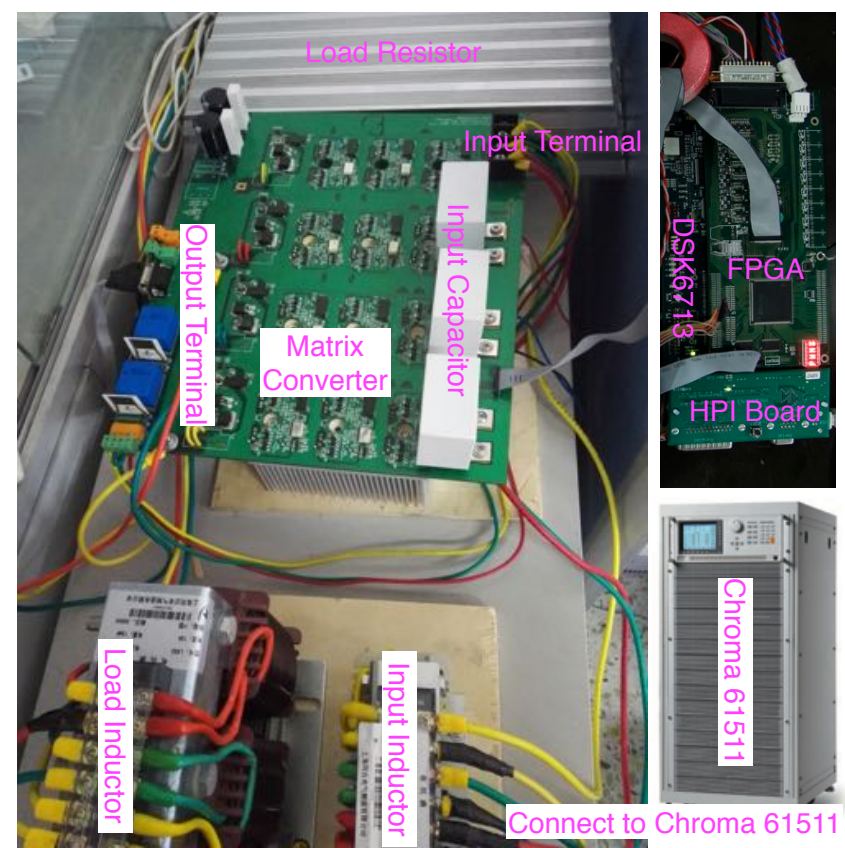

Fig. 11: The three-phase direct matrix converter prototype setups.

Table 3 System Parameters unless Otherwise Stated.

\begin{tabular}{l|c|c}
\hline \hline Variable & Description & Value \\
\hline$V_{i}$ & Supply Phase Voltage Amplitude & $155.5 \mathrm{~V}$ \\
$f_{i}$ & Input Fundamental Frequency & $50 \mathrm{~Hz}$ \\
$C_{f}$ & Input Filter Capacitor & $3 \mu \mathrm{F}$ \\
$L_{f}$ & Input Filter Inductor & $1 \mathrm{mH}$ \\
$f_{o}$ & Output Fundamental Frequency & $30 \mathrm{~Hz}$ \\
$R_{O}$ & Load Resistor & $50 \Omega$ \\
$L_{o}$ & Load Inductor & $15 \mathrm{mH}$ \\
$f_{s}$ & Sampling Frequency & $10 \mathrm{kHz}$ \\
IGBT & Insulated Gate Bipolar Transistor & SK80GM063 \\
DSP & Digital Signal Processor & TMS320C6713 \\
FPGA & Field Programmable Gate Array & A3P400 \\
$T_{d}$ & Commutation Step Delay & $0.4 \mu \mathrm{s}$ \\
\hline
\end{tabular}

memory interface of the DSP board to the FPGA which is responsible for implementing the four-step current commutation to generate the gate driving signals for IGBTs [43].

For the case of distorted input phase voltage, two dominant harmonics are injected into the fundamental component of $110 \mathrm{~V}$ root mean square (RMS) value. One is the 5 th order positive sequence harmonic with $0.07 p u$ amplitude, while the other is the $11 t h$ order negative sequence harmonic with $0.05 \mathrm{pu}$ amplitude. The waveforms of the input and output quantities when the modulation index $m$ is set to be constant 0.9 are shown in Fig. 12(a) and Fig. 12(b). As can be seen, without compensation, the input distortion affects the output voltage synthesis, and thus distorts the output currents. In more detail, the FFT analysis for the output current $I_{A}$ is given in Fig.12(c). Significant inter-harmonic contents around $300 \mathrm{~Hz}$ and $500 \mathrm{~Hz}$ can be observed. By contrast, Fig. 13(a) and Fig. 13(b) show the waveforms of the input and output variables after the input distortion is compensated. The output currents in Fig. 13(b) are much more sinusoidal with less distortion than those in Fig. 12(b). The RMS value of the output voltage is set as $80 \mathrm{~V}$ for fair comparisons. Further, the total harmonic distortion (THD) decreases from $6.21 \%$ to $4.00 \%$ after the compensation is actuated, as shown in Fig. 13(c). The inter-harmonics around the sideband of $300 \mathrm{kHz}$ have been suppressed.

For the case of unbalanced input phase voltages, the RMS value of phase "a" voltage is set to $121 \mathrm{~V}$ ( $10 \%$ higher than the other phases of $110 \mathrm{~V}$ RMS), while the output voltages maintain as $80 \mathrm{~V}$ RMS. The waveforms of the input quantities are shown in Fig. 14 while 


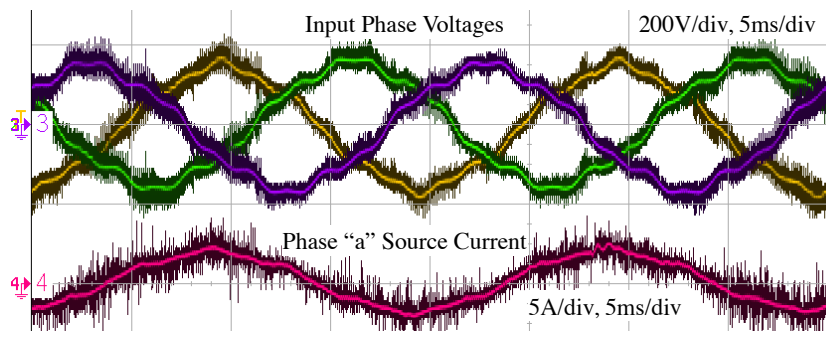

(a)

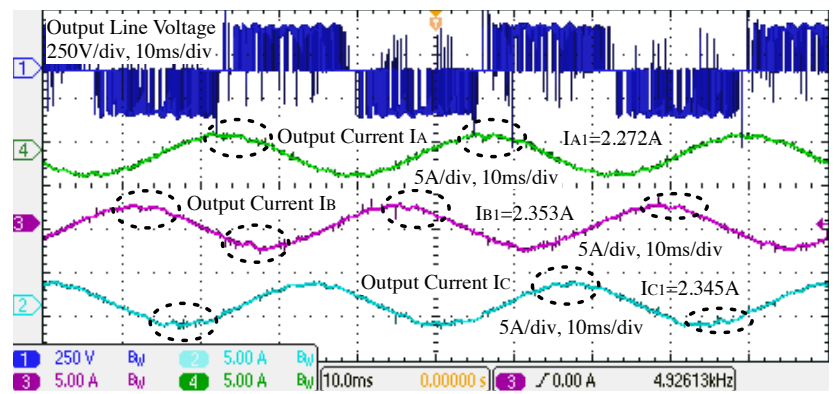

(b)

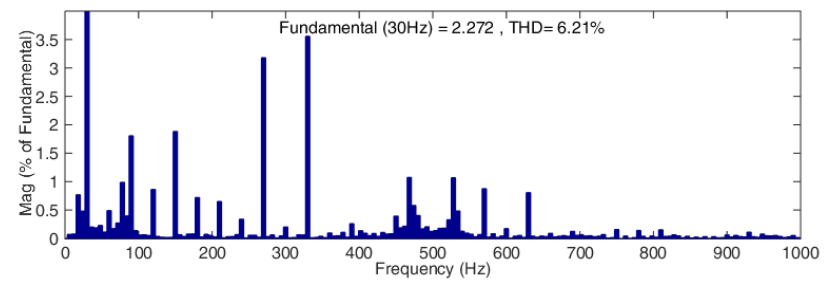

(c)

Fig. 12: The waveforms of the input/output quantities when the input distortion is uncompensated: (a) three input phase voltages and the source current $I_{a}$, (b) the output line-to-line voltage $v_{A B}$ and the three output currents, (c) the FFT analysis for the output current $I_{A}$.

those at the output side under two working conditions, i.e. with and without feedforward compensation, are shown in Fig. 15(a) and Fig. 15(b) respectively. The proposed ISVM method with feedforward compensation reduces the THD content to $3.32 \%$, whereas that in the standard ISVM method without feedforward capacity is $5.18 \%$. Also, the fundamental components of the three phase output currents are $2.14 \mathrm{~A}, 2.20 \mathrm{~A}$ and $2.18 \mathrm{~A}$, which means the output currents are balanced. Thus, the output current waveforms when the input unbalance is compensated are much smoother and have less THD than those when the input unbalance is uncompensated.

In both cases, the proposed SVD-based ISVM technique is able to offset the variations of the input voltages and keep the output currents sinusoidal and balanced. Such compensation is achieved by varying the modulation index according to the measured input voltages, as shown in Fig. 16. The THD contents of the A-phase output current $I_{A}$ with and without compensation operating under ideal, distorted or unbalanced input voltages are summarized in Table 4. In ideal scenarios when the input voltages are sinusoidal and balanced, both the input and output currents meet the quality requirements of IEEE Standard 519 [48]. In all conditions, the proposed ISVM has better output performance when the feedforward compensation capability is activated than not. However, if the input voltages are distorted or unbalanced the input currents will obviously become distorted. In such scenarios either the input or the output performance can be maintained leading to a sacrifice in the performance at the other side of the converter. It is worth noting that the input currents will be inevitably distorted if the output performance has a higher priority than that of the input side. This can be seen in Fig. 12(a), Fig. 13(a), Fig. 14(b) and Fig. 14(c), where the input current quality is slightly worse when the input voltages are compensated than those without compensation. Such phenomenon is intrinsically determined by the lack of energy storage elements in

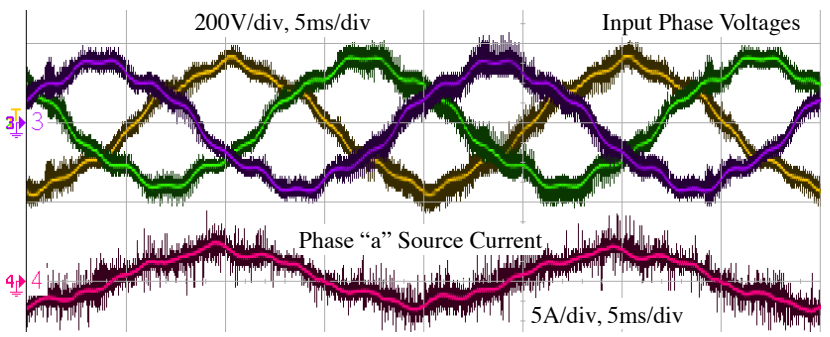

(a)

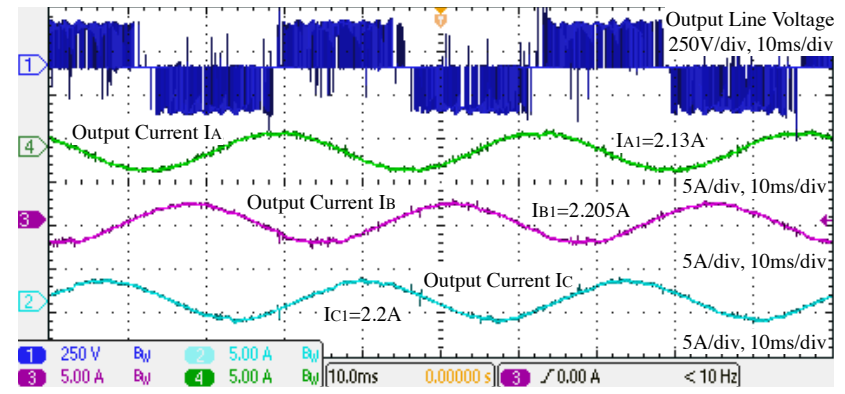

(b)

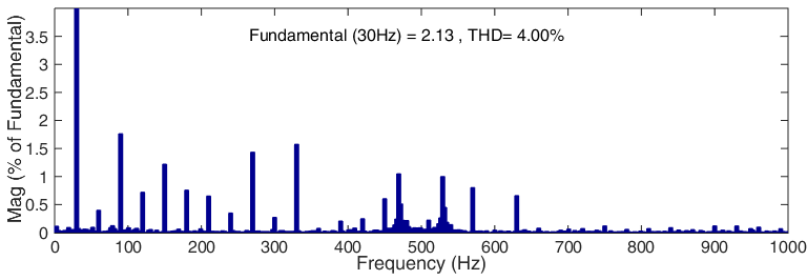

(c)

Fig. 13: The waveforms of the input/output quantities when the input distortion is compensated: (a) three input phase voltages and the source current $I_{a}$, (b) the output line-to-line voltage $v_{A B}$ and the three output currents, (c) the FFT analysis for the output current $I_{A}$.

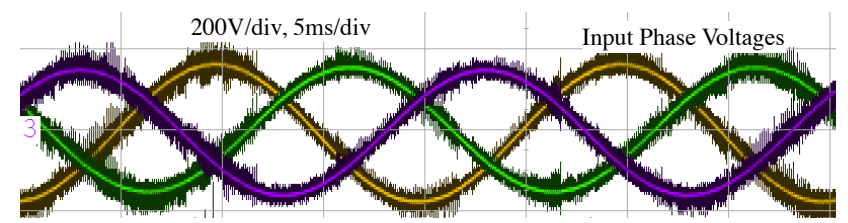

(a)

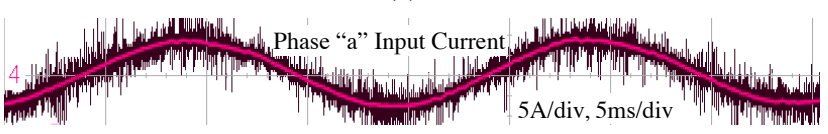

(b)

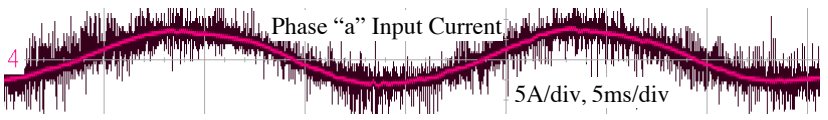

(c)

Fig. 14: The waveforms of the input side quantities: (a) three unbalanced input phase voltages, (b) the source current $I_{a}$ when the input imbalance is uncompensated, (c) the source current $I_{a}$ when the input imbalance is compensated.

matrix converters and the instantaneous active power balance theory [47]. Additional effort in hardware or software can change this compromise [44-47], but these are beyond the scope of this paper.

The proposed method has similar performance under other abnormal supply conditions, like sags, surges, and thus the corresponding results are not shown here. 


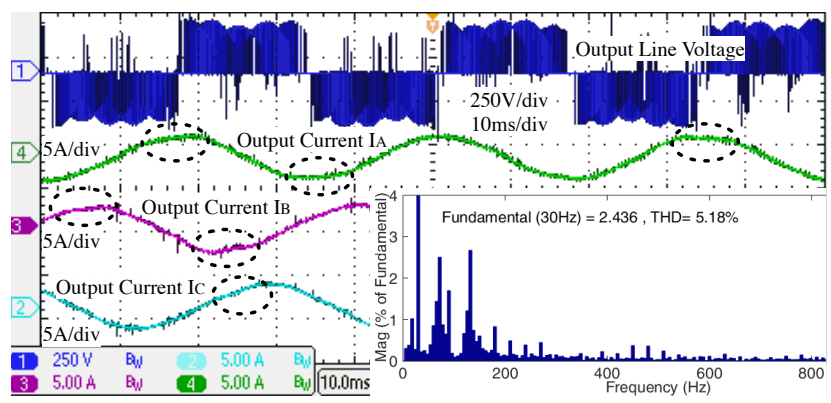

(a)

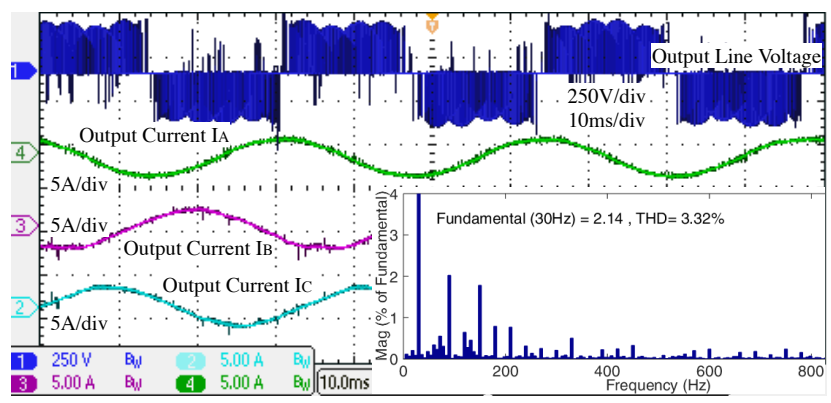

(b)

Fig. 15: The waveforms of the output voltage and currents when the unbalanced input voltages are (a) uncompensated, and (b) compensated. From top to bottom: the output line-to-line voltage $v_{A B}$ and the three output currents. The floating subfigure is the FFT analysis for the phase-A output current $I_{A}$.

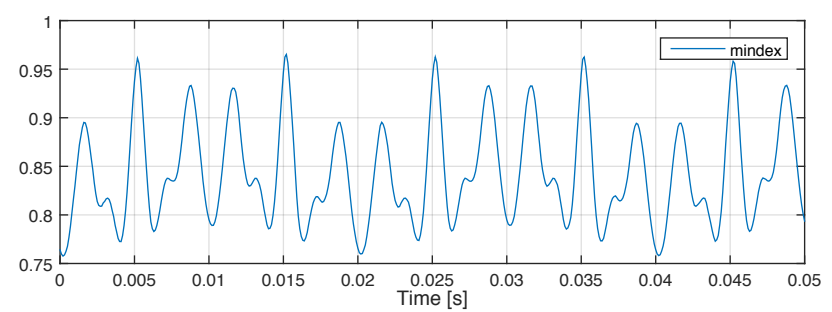

(a)

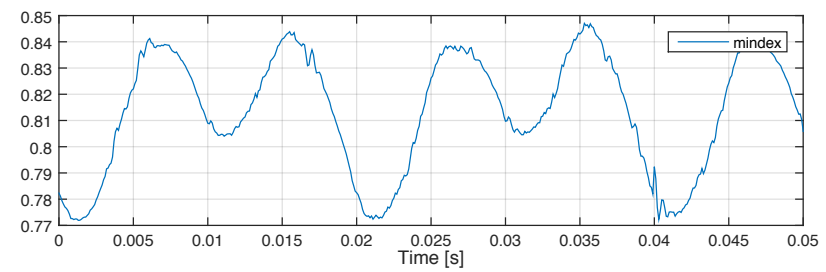

(b)

Fig. 16: Varying modulation index $m$ to compensate the abnormal supply conditions: (a) distorted input, (b) unbalanced input voltages.

Table 4 THD summary under different input voltage conditions

\begin{tabular}{l|c|c|c}
\hline \hline THD of Output Current $I_{A}$ & Ideal & Distorted & Unbalanced \\
\hline Uncompensated & $1.86 \%$ & $6.21 \%$ & $5.18 \%$ \\
\hline Compensated & $1.53 \%$ & $4.00 \%$ & $3.32 \%$ \\
\hline \hline THD of Intput Current $I_{a}$ & Ideal & Distorted & Unbalanced \\
\hline Uncompensated & $1.94 \%$ & $12.83 \%$ & $8.69 \%$ \\
\hline Compensated & $2.17 \%$ & $17.22 \%$ & $9.91 \%$ \\
\hline
\end{tabular}

By setting a counter in the program, the maximum execution time in our DSP platform is $83.0 \mu \mathrm{s}$, which is almost the same as that for the traditional ISVM without compensating the abnormal input voltages, $82.8 \mu \mathrm{s}$.

\section{Conclusions}

This paper has presented a new indirect space vector modulation (SVM) with feedforward compensation mechanism for both the direct and indirect matrix converters. The singular value decomposition (SVD) technique is applied on the switch states to reveal the intrinsic features of matrix converters. A duty-cycle matrix is then constructed using the SVD results of switch states. The SVD also renders the switch states and the modulation process a geometrical interpretation between the system input and output vectors. To implement the proposed modulation construction, the space vector synthesis technique is subsequently utilized to select a series of switch states and calculate their duty cycles in a simple way. By including the instantaneous input voltages in the calculations, the duty cycles are adjusted on-line to counteract the undesirable effects caused by abnormal input voltages to keep the output currents sinusoidal and balanced. Combining SVD and SVM provides the modulation process of matrix converters a geometrical visualization, and hence a measure not only to revisit the existing modulation methods but also to design new ones. Moreover, it provides a flexibility in terms of switching state selection, duty-cycle calculation and switching sequence arrangement, making the modulation easy to understand and implement. The experimental results have been shown to substantiate the claims above.

\section{References}

1 P. Wheeler, J. Rodriguez, J. Clare, L. Empringham, and A. Weinstein, "Matrix converters: a technology review," IEEE Trans. Industrial Electronics, vol. 49, no. 2, pp. 276-288, 2002.

2 L. Empringham, J. Kolar, J. Rodriguez, W. Patrick, and J. Clare, "Technological issues and industrial application of matrix converters: A review," IEEE Trans. Industrial Electronics, vol. 60, no. 10, pp. 4260-4271, 2013.

3 M. Aten, G. Towers, C. Whitley, P. Wheeler, J. Clare, and K. Bradley, "Reliability comparison of matrix and other converter topologies," IEEE Trans. Aerospace and Electronic Systems, vol. 42, no. 3, pp. 867-875, 2006.

4 O. Simon, J. Mahlein, M. Muenzer, and M. Bruckmarm, "Modern solutions for industrial matrix-converter applications," IEEE Trans. Industrial Electronics, vol. 49, no. 2, pp. 401-406, 2002.

5 A. Garcés and M. Molinas, "Coordinated control of series-connected offshore wind park based on matrix converters," Wind Energy, vol. 15, no. 6, pp. 827-845, 2012.

6 A. Garces and M. Molinas, "A study of efficiency in a reduced matrix converter for offshore wind farms," Industrial Electronics, IEEE Transactions on, vol. 59, pp. $184-193$, Jan. 2012.

7 J. E. Huber and J. W. Kolar, "Solid-State Transformers: On the Origins and Evolution of Key Concepts," IEEE Industrial Electronics Magazine, vol. 10, pp. 19-28, Sept. 2016.

8 U. Nasir, A. Costabeber, P. Wheeler, M. Rivera, and J. Clare, "A Three-Phase Modular Isolated Matrix Converter," IEEE Trans. Power Electronics, pp. 1-1, 2019.

9 D. Chen and T. Liu, "Implementation of a novel matrix converter PMSM drive," IEEE Trans. Aerospace and Electronic Systems, vol. 37, no. 3, pp. 863-875, 2001. 
10 T.-H. Liu, S.-H. Chen, and D.-F. Chen, "Design and implementation of a matrix converter PMSM drive without a shaft sensor," IEEE Trans. Aerospace and Electronic Systems, vol. 39, pp. 228-243, Jan. 2003.

11 S. Lopez Arevalo, P. Zanchetta, P. Wheeler, A. Trentin, and L. Empringham, "Control and Implementation of a Matrix-Converter-Based AC Ground Power-Supply Unit for Aircraft Servicing," IEEE Trans. Industrial Electronics, vol. 57, pp. 2076 -2084, June 2010.

12 W. Rohouma, P. Zanchetta, P. Wheeler, and L. Empringham, "A Four-Leg Matrix Converter Ground Power Unit With Repetitive Voltage Control," IEEE Trans. Industrial Electronics, vol. 62, pp. 2032-2040, Apr. 2015.

13 J. Rodriguez, M. Rivera, J. Kolar, and P. Wheeler, "A review of control and modulation methods for matrix converters," IEEE Trans. Industrial Electronics, vol. 59, no. 1, pp. 58-70, 2012.

14 P. Kiatsookkanatorn and S. Sangwongwanich, "A unified PWM method for matrix converters and its carrier-based realization using dipolar modulation technique," IEEE Trans. Industrial Electronics, vol. 59, no. 1, pp. 80-92, 2012.

15 D. Casadei, G. Serra, A. Tani, and L. Zarri, "Matrix converter modulation strategies: a new general approach based on space-vector representation of the switch state," IEEE Trans. Industrial Electronics, vol. 49, no. 2, pp. 370-381, 2002.

16 H. Hojabri, H. Mokhtari, and L. Chang, "A generalized technique of modeling, analysis, and control of a matrix converter using SVD," IEEE Trans. Industrial Electronics, vol. 58, no. 3, pp. 949-959, 2011.

17 L. C. Gili, L. O. Seman, and S. V. G. Oliveira, "Different Switching Sequence Comparison Applied to Multimodular Matrix Converter using ISVM," IEEE Latin America Transactions, vol. 16, pp. 1595-1602, June 2018.

18 H.-N. Nguyen and H.-H. Lee, "An Enhanced SVM Method to Drive Matrix Converters for Zero CommonMode Voltage," IEEE Trans. Power Electronics, vol. 30, no. 4, pp. 1788-1792, 2015.

19 H.-N. Nguyen and H.-H. Lee, "A DSVM method for matrix converters to suppress common-mode voltage with reduced switching losses," IEEE Trans. Power Electronics, vol. 31, no. 6, pp. 4020-4030, 2016.

20 H. N. Nguyen and H. H. Lee, "A Modulation Scheme for Matrix Converters With Perfect Zero CommonMode Voltage," IEEE Trans. Power Electronics, vol. 31, pp. 5411-5422, Aug. 2016.

21 A. Tsoupos and V. Khadkikar, "A Novel SVM Technique With Enhanced Output Voltage Quality for Indirect Matrix Converters," IEEE Trans. Industrial Electronics, vol. 66, pp. 832-841, Feb. 2019.

22 H. Wang, Y. Zhang, M. Su, Y. Sun, X. Li, and G. Zhang, "Control method for the two-stage matrix converter to enhance the linear voltage transfer ratio," IET Power Electronics, vol. 11, no. 14, pp. 2295-2301, 2018.

23 Y. Xia, X. Zhang, M. Qiao, F. Yu, Y. Wei, and P. Zhu, "Research on a New Indirect Space-Vector Overmodulation Strategy in Matrix Converter," IEEE Trans. Industrial Electronics, vol. 63, pp. 1130-1141, Feb. 2016.
24 L. Huber and D. Borojevic, "Space vector modulated three-phase to three-phase matrix converter with input power factor correction," IEEE Trans. Industry Applications, vol. 31, no. 6, pp. 1234-1246, 1995.

25 M. Jussila and H. Tuusa, "Comparison of simple control strategies of space-vector modulated indirect matrix converter under distorted supply voltage," IEEE Trans. Power Electronics, vol. 22, no. 1, pp. 139-148, 2007.

26 J. W. Kolar, F. Schafmeister, S. D. Round, and H. Ertl, "Novel three-phase AC-AC sparse matrix converters," IEEE Trans. Power Electronics, vol. 22, no. 5, pp. 16491661, 2007.

27 J. Dasika and M. Saeedifard, "An Online Modulation Strategy to Control the Matrix Converter Under Unbalanced Input Conditions," IEEE Trans. Power Electronics, vol. 30, no. 8, pp. 4423-4436, 2015.

28 L. Wei, Y. Matsushita, and T. Lipo, "A compensation method for dual-bridge matrix converters operating under distorted source voltages," in Proc. IEEE Industrial Electronics Society Annu. Conf. (IECON'03), vol. 3, pp. 2078-2084 Vol.3, 2003.

29 L. Wei, Y. Matsushita, and T. Lipo, "Investigation of dual-bridge matrix converter operating under unbalanced source voltages," in Proc. IEEE 34th Annual Power Electronics Specialist Conf. (PESC'03), vol. 3, pp. 1293 1298, June 2003.

30 X. Xu, M. Su, Y. Sun, and W. Gui, "Two new modulation strategies for two-stage matrix converter under nonsinusoidal input voltages," in Proc. 2nd IEEE Conf. Industrial Electronics and Applications (ICIEA'07), pp. 19171921, 2007.

31 P. Nielsen, F. Blaabjerg, and J. Pedersen, "Space vector modulated matrix converter with minimized number of switchings and a feedforward compensation of input voltage unbalance," in Proc. Int. Conf. Power Electronics, Drives and Energy Systems for Industrial Growth, vol. 2, pp. 833-839, 1996.

32 H. She, H. Lin, X. Wang, and S. Xiong, "Space vector modulated matrix converter under abnormal input voltage conditions," in Proc. 6th IEEE Int. Power Electronics and Motion Control Conf. (IPEMC'09), pp. 1723-1727, 2009.

33 V. Vekhande, B. Pimple, and B. Fernandes, "Modulation of indirect matrix converter under unbalanced source voltage condition," in Proc. IEEE Energy Conversion Congress and Exposition (ECCE '11), pp. 225 -229, Sept. 2011.

34 X. Wang, H. Lin, H. She, and B. Feng, "A research on space vector modulation strategy for matrix converter under abnormal input-voltage conditions," IEEE Trans. Industrial Electronics, vol. 59, pp. 93 -104, Jan. 2012.

35 O. Simon and M. Braun, "Theory of vector modulation for matrix converters," in Proc. EPE '01, pp. 1-7, 2001.

36 Q. Guan, P. Yang, Q. Guan, and X. Wang, "SVD-based indirect space vector modulation with feedforward compensation for matrix converters," in Proc. 39th Annual Conference of the IEEE Industrial Electronics Society (IECON'13), pp. 4955-4960, 2013.

37 Q. Guan, P. Wheeler, Q. Guan, and P. Yang, "Commonmode Voltage Reduction for Matrix Converters Using 
All Valid Switch States," IEEE Trans. Power Electronics, vol. 31, no. 12, pp. 8247-8259, 2016.

38 Q. Guan, P. Yang, Q. Guan, X. Wang, and Q. Wu, "A singular value decomposition-based space vector modulation to reduce the output common-mode voltage of direct matrix converters," Journal of Power Electronics, vol. 16, no. 3, pp. 936-945, 2016.

39 Q. Guan, X. Wang, Z. Zhao, and L. Wang, "An SVDbased SVM with common-mode voltage reduction for direct matrix converters," in Proc. 9th International Conference on Power Electronics-ECCE Asia (ICPE-ECCE Asia), (Soul, Korea), pp. 2697-2700, 2015.

40 E. Un and A. Hava, "A near-state PWM method with reduced switching losses and reduced common-mode voltage for three-phase voltage source inverters," IEEE Trans. Industry Applications, vol. 45, no. 2, pp. 782-793, 2009.

41 A. Hava and E. Un, "A high-performance PWM algorithm for common-mode voltage reduction in threephase voltage source inverters," IEEE Trans. Power Electronics, vol. 26, no. 7, pp. 1998-2008, 2011.

42 A. D. Arioni, T. S. Padilha, and S. V. G. Oliveira, "Expanded Space Vector Modulation of Direct Matrix Converters Including Hidden Rotating Pairs," IEEE Trans. Ind. Electron., vol. 66, no. 11, pp. 8296-8307, Nov. 2019.

43 P. Wheeler, J. Clare, and L. Empringham, "Enhancement of matrix converter output waveform quality using minimized commutation times," IEEE Trans. Industrial Electronics, vol. 51, no. 1, pp. 240-244, 2004.

44 D. Casadei, G. Serra, A. Tani, and L. Zarri, "Stability analysis of electrical drives fed by matrix converters," Proc. IEEE Int. Symp. Industrial Electronics, ISIE'02, vol. 4, pp. 1108-1113, 2002.

45 D. Casadei, G. Serra, A. Tani, A. Trentin, and L. Zarri, "Theoretical and experimental investigation on the stability of matrix converters," IEEE Trans. Industrial Electronics, vol. 52, no. 5, pp. 1409-1419, 2005.

46 D. Casadei, J. Clare, L. Empringham, G. Serra, A. Tani, A. Trentin, P. Wheeler and L. Zarri, "Large-Signal model for the stability analysis of matrix converters," IEEE Trans. Industrial Electronics, vol. 54, no. 2, pp. 939-950, 2007.

47 Q. Guan, P. Yang, X. Wang, and X. Zhang, "Stability analysis of matrix converter with constant power loads and LC input filter," in Proc. 7th IEEE Int. Power Electronics and Motion Control Conf. (IPEMC'12), vol. 2, pp. $900-904,2012$.

48 "IEEE Recommended Practice and Requirements for Harmonic Control in Electric Power Systems," IEEE STD. 519-2014, 27 March 2014, 